Ann. Biol. anim. Bioch. Biophys., I974, 14 (4-B), 793-8II.

\title{
ÉVOLUTION DES RÉSERVES LIPIDIQUES DU JEUNE RAT EN GROISSANCE EN FONCTION DU TEMPS ET DE LA QUALITÉ DES LIPIDES INGÉRÉS
}

\author{
Y. DEMARNE, Maïmouna TOURÉ, Françoise DESNOYERS, \\ N. VODOVAR et J. FLANZY
}

avec la collaboration technique de Marie-José Lecourtier et S. Delpai

Station de Recherches de Nutrition, Centre national de Recherches zootechniques, I. N.R. A., 78350 Jouy en Josas

\section{RÉSUMÉ}

La teneur en lipides totaux, la composition en acides gras des lipides, des carcasses et des foies ainsi que la structure histologique du tissu adipeux mésentérique sont suivies pendant 6 semaines dans 2 lots de jeunes rats récemment sevrés. Les animaux reçoivent le même régime de base et absorbent des quantités isoénergétiques de lipides contenant soit 7o p. roo d'acides gras longs saturés (autant de $\mathrm{C}_{16: 0}$ que de $\mathrm{C}_{18: 0}$ ) soit $85 \mathrm{p}$. roo d'acides gras longs et insaturés $\left(\mathrm{C}_{18: 1}\right.$ et $\left.\mathrm{C}_{18: 2}\right)$.

Dans les conditions expérimentales précisées dans le texte, la croissance, l'adipogenèse et la protéinogenèse sont identiques dans les 2 lots.

La composition en acides gras des réserves corporelles ainsi que celle des lipides hépatiques est totalement modifiée après 7 jours de soumission aux 2 régimes expérimentaux et les compositions en acides gras sont considérées comme étant totalement stabilisées au bout de 2 I jours. Au niveau des lipides corporels, l'absorption d'acides gras saturés et longs $\left(\mathrm{C}_{16: 0}\right.$ et $\left.C_{18: 0}\right)$ favorise la mise en réserve d'une forte quantité d'acides gras mono-insaturés et principalement d'acide oléique $\left(\mathrm{C}_{18: 1}\right)$.

En cas d'un fort apport exogène d'acides gras saturés et longs (autant de $\mathrm{C}_{\mathbf{1 6}: \mathbf{0}}$ que de $\left.\mathrm{C}_{18: 0}\right)$ ou en cas de fort apport d'acides gras longs mono- et poly-insaturés $\left(\mathrm{C}_{18: 1}\right.$ et $\left.\mathrm{C}_{18: 2}\right)$ on ne modifie que très peu le rapport $\Sigma$ acides gras saturés/ $\Sigma$ acides gras insaturés dans les lipides corporels. En revanche, on modifie sérieusement le rapport acide oléique déposé/ $\Sigma$ des acides gras insaturés déposés. Ces résultats soulignent donc le rôle joué par l'acide oléique dans la régulation du rapport $\Sigma$ acides gras saturés $/ \Sigma$ acides gras insaturés en fonction du type de lipide ingéré.

Les études histologiques montrent que dans nos conditions d'alimentation et de croissance, et jusqu'à l'âge d'environ 70 jours, on n'observe aucune différence morphologique entre les deux types de tissu adipeux mésentérique. 


\section{INTRODUCTION}

De très nombreux travaux montrent que la composition en acides gras des réserves adipeuses de l'animal monogastrique est très fortement influencée par celle des lipides ingérés (TOve et SMith, r960; Kirschner et Harris, I96I). Le phénomène est particulièrement marqué lorsque l'ingestion d'un type particulier de lipide se prolonge, par exemple, pendant toute la durée de la croissance de l'animal (CARROL, , Ig65; CLÉMENT et al., I965).

Cependant, peu de travaux abordent l'étude de l'évolution de la composition en acides gras des lipides corporels en fonction du type de lipide ingéré pendant les premières semaines qui suivent le sevrage.

Notre but dans ce travail a donc été l'étude systématique de l'évolution en fonction du temps et du régime alimentaire, de la composition en acides gras des lipides des réserves adipeuses et du tissu hépatique, chez de jeunes rats en croissance récemment sevrés, recevant soit un régime très riche en acides gras longs et saturés $\left(\geqslant \mathrm{C}_{16}: 0\right)$, soit un régime très riche en acides gras longs mono- et poly-insaturés $\left(\mathrm{C}_{18: 1 \omega 9}\right.$ et $\left.\mathrm{C}_{18: 2 \omega_{6}}\right)$.

Conjointement aux études biochimiques, des observations morphologiques concernant l'évolution des cellules adipeuses ont été faites sur les animaux soumis aux deux régimes.

\section{MÉTHODES}

\section{I. - Régimes}

Les matières grasses utilisées sont le beurre de cacao et l'huile de tournesol (voir composition sur le tableau $\mathrm{r}$ ).

L'expérimentation animale ayant pour but l'étude comparative des effets lipogénétiques des acides gras longs et saturés et des acides gras insaturés présente certaines difficultés. Ainsi, le fait que les animaux acceptent mal les régimes comportant des lipides très saturés dans leur ration et réduisent leur consommation alimentaire, joint au fait que leurs utilisations digestives (CUDa) sont très basses alors que celles des lipides insaturés sont élevées, font qu'il est très difficile d'assurer pour 2 lots différents d'animaux un même apport quotidien de lipides digestibles avec ces deux types de matières grasses.

Le problème est résolu à l'aide d'une première expérimentation réalisée avec 2 lots de 6 ratons de souche Wistar recevant le même régime de base que celui devant être utilisé par la suite (ro p. roo de beurre de cacao ou d'huile de tournesol), et qui permet:

- de déterminer le CUDa des lipides testés ainsi que ceux de leurs acides gras ;

- de s'assurer que les absorptions apparentes des protéines $(N \times 6,25)$ et du reste de la ration ne sont pas modifiées par la qualité des lipides ingérés;

- de déterminer enfin le seuil d'acceptation du régime contenant le beurre de cacao.

Quelques-uns des principaux résultats de cette pré-expérimentation sont rapportés dans le tableau 2.

On procède ensuite à la préparation du régime semi-synthétique " $T$ " dont la composition est rapportée dans le tableau 3 et qui contient une quantité $\mathrm{X}$ d'huile de tournesol. Puis on prépare le régime " $\mathrm{C}$ " contenant une quantité $\mathrm{Y}$ de beurre de cacao, $\mathrm{Y}$ étant lié à $\mathrm{X}$ par le rapport des deux CUDa, soit $\mathrm{Y}=\mathrm{X} \times \frac{95}{65}$. Les quantités des autres éléments introduits dans les rations sont identiques dans les 2 cas. 
TABLEAU I

Composition en acides gras des lipides ingérés

(p. roo des esters méthyliques dosés)

\begin{tabular}{c|c|c|c|c}
\hline \hline Régimes & $\mathrm{C}_{\mathbf{1 6}}: 0$ & $\mathrm{C}_{\mathbf{1 8}}: \mathbf{0}$ & $\mathrm{C}_{\mathbf{1 8}: 1}$ & $\mathrm{C}_{\mathbf{1 8}}: \mathbf{2}$ \\
\hline $\mathrm{C}$ & 32,5 & 38,1 & $-26,1$ & 3,3 \\
\hline $\mathrm{T}$ & 6,5 & 6,5 & 23,4 & 62,6 \\
\hline
\end{tabular}

\section{TABLEAU 2}

Principaux résultats de l'étude pré-expérimentale.

Utilisations digestives apparentes CUDa des principaux éléments de la ration (valeurs moyennes)

\begin{tabular}{|c|c|c|c|c|}
\hline Régimes & $\begin{array}{c}\text { CUDa MS* } \\
(\%)\end{array}$ & $\begin{array}{c}\text { CUDa MG* } \\
(\%)\end{array}$ & $\begin{array}{c}\text { CUDa P* } \\
(\%)\end{array}$ & $\begin{array}{c}\text { CUDa MSL* } \\
(\%)\end{array}$ \\
\hline C & 94,0 & 95,0 & 92,4 & $9 !, 0$ \\
\hline $\mathrm{T}$ & 91,5 & 65,0 & 93,8 & 94,9 \\
\hline
\end{tabular}

* MS : Matière sèche ; $M G$ : Lipides; $\mathrm{P}$ : protéines $(\mathrm{N} \times 6,25)$; MSL : fraction non lipidique de la ration.

\section{TABLEAU 3}

Composition des régimes expérimentaux $C$ et $T$

\begin{tabular}{|c|c|c|c|c|}
\hline & \multicolumn{2}{|c|}{$\begin{array}{c}\text { Régime " Huile de Tournesol » } \\
\text { T }\end{array}$} & \multicolumn{2}{|c|}{$\begin{array}{l}\text { Régime " Beurre de Cacao " } \\
\text { C }\end{array}$} \\
\hline & quantité (g) & pourcentage & quantité (g) & pourcentage \\
\hline Caséine + A. A. complémentaire & 370 & 18,5 & 370 & 17,7 \\
\hline Amidon de maïs . ........... & 870 & 43,5 & 870 & 41,6 \\
\hline Sucre............... & 430 & 21,5 & 430 & 20,6 \\
\hline Agar-Agar $\ldots \ldots \ldots \ldots \ldots$ & 54 & 2,7 & 54 & 2,6 \\
\hline Minéraux + vitamines $\ldots \ldots \ldots$. & 76 & 3,8 & 76 & 3,6 \\
\hline Total $\ldots \ldots \ldots \ldots \ldots$ & 1800 & 90,0 & 1800 & 86,1 \\
\hline $\begin{array}{l}\text { Huile de Tournesol } \ldots \ldots \ldots \ldots \\
\text { Beurre de Cacao } \ldots \ldots \ldots \ldots\end{array}$ & $\begin{array}{l}X=200 \\
-\end{array}$ & 10,0 & $\mathrm{Y}=290^{*}$ & $\overline{13,9}$ \\
\hline Total & 2000 & 100,0 & 2090 & 100,0 \\
\hline
\end{tabular}

$* \mathrm{Y}=\frac{\mathrm{X} \times 95}{65}$. 
Puisque, dans nos conditions expérimentales, le type de matière grasse n'influence pas le CUDa des protéines $(\mathrm{N} \times 6,25)$, ni celui du reste de la ration, lorsqu'un animal recevant le régime " $C$ " ingère QC grammes d'aliment $C$, il faut que son homologue recevant le régime $T$ ingère $Q T$ grammes d'aliment $T$ pour que les apports de lipides digestibles et des autres constituants de la ration soient identiques dans les 2 cas, $Q T$ étant lié à $Q \mathrm{C}$ par la relation :

$$
\mathrm{QT}=\mathrm{QC} \times \frac{2000}{2090}=\mathrm{QC} \times 0,957
$$

\section{2. - Conduite de l'expérience}

L'expérience est réalisée avec 48 rats mâles de souche Wistar récemment sevrés et provenant de la même animalerie (alimentation identique pour toutes les mères). Les animaux sont répartis en 2 lots " $\mathrm{C}$ " et " $\mathrm{T}$ " homogènes de 24 rats et le poids moyen en début d'expérimentation est de $65,5 \mathrm{~g}$.

L'expérience se déroule dans une raterie conditionnée (température, hygrométrie, lumière) et les animaux sont disposés en cages individuelles.

Au premier jour de l'experience, 4 ratons sont choisis au hasard ( 2 dans chaque lot) et sacrifiés. On prélève aussitôt sur ces animaux des échantillons de tissu adipeux mésentérique afin de procéder aux analyses histologiques et on détermine la composition corporelle des animaux abattus. On procède également à la détermination de la composition en acides gras des lipides corporels et hépatiques.

Par la suite, les sacrifices, prélèvements et analyses sont régulièrement répétés aux $7^{\mathrm{e}}, \mathrm{I}_{4}^{\mathrm{e}}$, $2 \mathrm{r}^{\mathrm{e}}, 28^{\mathrm{e}}, 35^{\mathrm{e}}$ et $42^{\mathrm{e}}$ jour de l'expérience. A chaque fois, 4 animaux de chaque lot sont sacrifiés.

Les animaux reçoivent une ration alimentaire quotidienne calculée selon les critères présentés au paragraphe précédent. Ce rationnement, déterminé en fonction de la quantité maxima moyenne d'aliment acceptée dans le lot "C ", permet d'ajuster au mieux les rations dans les deux cas. L'expérience débute à un niveau d'ingestion quotidien moyen de 9 grammes de matière sèche par rat dans le lot $\mathrm{C}$. En fonction de la croissance des animaux, le volume de la ration est progressivement augmenté, en évitant au maximum la formation de refus dans le lot " $\mathrm{C}$ ». Ce mode d'alimentation permet d'obtenir une croissance rectiligne située aux environs de $4 \mathrm{~g} / \mathrm{jour}$ dans les deux lots.

\section{3. - Méthodes d'analyse}

Après abattage on procède immédiatement au prélèvement et à la pesée du foie. Le reste de la carcasse est plongé dans l'azote liquide puis broyé dans un hachoir à viande (type Scharfen). Les carcasses broyées sont ensuite lyophilisées.

Les teneurs en eau sont estimées par différence de poids avant et après lyophilisation. Les teneurs en matière azotée totale $(\mathrm{N} \times 6,25)$ sont déterminées par dosage de l'azote selon la méthode de Kjeldahl.

Les lipides totaux des carcasses et des foies sont extraits à froid en suivant la technique de Folch, Lees et Sloane Stanley (1957). Les acides gras sont séparés des autres constituants lipidiques par saponification et ils sont identifiés et dosés par chromatographie en phase gazeuse après avoir été méthylés.

Les conditions d'analyse sont les suivantes : appareil Microtek GC $2500 \mathrm{R}$; longueur de la colonne : $3 \mathrm{~m}$; diamètre : 1,8 inch ; phase stationnaire EGSS (5 p. Ioo) sur Chromosorb GDMCS $80 / 100$ mesh ; gaz vecteur : azote; $t$ colonne : $170^{\circ} \mathrm{C}$; nombre de plateaux théoriques : environ 2500 ; détection à ionisation de flamme et intégration électromécanique.

\section{4. - Prélèvement et préparation des tissus pour les observations morphologiques}

Les fragments de tissu adipeux mésentérique, dont l'aspect structural et ultrastructural est susceptible d'être influencé, dans certaines conditions d'expérience, par les lipides du régime ont été prélevés sur deux rats sacrifiés au moment de la mise en expérience, puis sur deux rats de chaque lot " $\mathrm{C}$ " et " $\mathrm{T}$ " après 7 jours d'expérience, puis tous les 7 jours jusqu'à 42 jours. Sur les animaux endormis, les tissus adipeux mésentériques d'un niveau choisi sont fixés in situ pendant 5 à ro minutes avec le tétroxyde d'osmium à $2 \mathrm{p}$. Ioo tamponné phosphate à $\mathrm{pH} 7,2$ selon MiL. LONIG (I96r), puis après extirpation dans le même fixateur pendant is heures à $4^{\circ} \mathrm{C}$. Après lavage dans le tampon, déshydratation avec l'acétone et une inclusion dans le mélange d'Épon 8I 2, les blocs sont coupés avec l'ultratome LKB III puis contrastés avec l'acétate d'uranyl et le citrate 
de plomb et observés avec l'Elmiscop IA. Des coupes semi fines de $2 \mu$ ont été réalisées, sur les tissus préparés pour la microscopie électronique, avec le Pyramitome LKB, colorées suivant la technique de LEEson et LEEson (1970) et observées au microscope photonique Orthoplan Leitz.

\section{RÉSULTATS}

I. - Évolution de la composition en acides gras des lipides corporels en fonction du temps

Les bilans nutritionnels, la croissance et l'évolution de la composition des carcasses sont suivis pendant 4 semaines. Malgré les nombreuses difficultés inhérentes à la nature même de l'expérience, on assure un apport de protéines et de lipides digestibles similaire dans les 2 lots. Les animaux des 2 lots absorbent en moyenne et mettent en réserve des quantités identiques de lipides (tabl. 4).

TABLEAU 4

Croissance et bilan nutritionnel

(valeurs moyennes exprimées en grammes par jour)

\begin{tabular}{|c|c|c|c|c|c|c|}
\hline Régimes & Croissance & $\begin{array}{l}\text { Lipides } \\
\text { absorbés }\end{array}$ & $\begin{array}{c}\text { Protéines } \\
\text { absorbées* }\end{array}$ & $\begin{array}{l}\text { Lipides } \\
\text { déposés }\end{array}$ & $\begin{array}{l}\text { Protéines } \\
\text { déposées }\end{array}$ & $\begin{array}{c}\text { Stockage } \\
\text { apparent } \\
\text { des lipides } \\
(\%)\end{array}$ \\
\hline$T$ & 3,93 & 0,93 & 1,48 & 0,41 & 0,82 & 44,09 \\
\hline $\mathrm{C}$ & 3,71 & 0,99 & 1,57 & 0,41 & 0,74 & 42,42 \\
\hline
\end{tabular}

$* N \times 6,25$.

Les tableaux 5 et 6 et les graphiques I et 2 rapportent l'évolution de la composition en acides gras des lipides corporels en fonction du temps et du traitement.

Après 7 jours de mise aux régimes $\mathrm{C}$ et $\mathrm{T}$, on observe un profond remaniement de la composition en acides gras des réserves adipeuses.

Dans les 2 lots, on note la disparition des acides gras à chaines moyennes $C_{10}$, $\mathrm{C}_{12}$ et $\mathrm{C}_{14}$ (présents en quantités non négligeables dans le lait de Rate, SMrTH, WaTTs et DILs, rg68).

Les autres remaniements observés diffèrent en fonction du régime testé :

- En cas d'ingestion de beurre de cacao (lot $\mathrm{C}$ ) on remarque une importante diminution de la concentration en acide linoléique $\left(C_{18: 2}\right)$, une très forte augmentation de la concentration en acide oléique $\left(\mathrm{C}_{18}: 1\right)$ et $\mathrm{un}$ maintien des concentrations respectives en acide palmitique $\left(C_{16: 0}\right)$, palmitoléique $\left(C_{16: 1}\right)$ et stéarique $\left(C_{18: 0}\right)$. 
- En cas d'ingestion d'huile de tournesol (lot 'T) on observe une augmentation de la concentration en acide linoléique $\left(\mathrm{C}_{18: 2}\right)$, une diminution de la concentration en acide palmitique $\left(\mathrm{C}_{16: 0}\right)$ et un maintien des concentrations des autres acides gras.

- Par la suite, on note de très légères modifications des compositions en acides gras des réserves lipidiques, mais qui ne prennent jamais l'ampleur de celles qui sont observées pendant la première semaine.

\section{TABLEAU 5}

Évolution de la composition en acides gras des lipides corporels des rats ayant ingéré le régime $T$ "Huile de Tournesol"

(p. roo des esters méthyliques dosés)

\begin{tabular}{c|c|c|c|c|c|c|c|c|c}
\hline \hline$t(\mathrm{j})$ & $\mathrm{C}_{10}$ & $\mathrm{C}_{12}$ & $\mathrm{C}_{14}$ & $\mathrm{C}_{16}$ & $\mathrm{C}_{16: 1}$ & $\mathrm{C}_{18}$ & $\mathrm{C}_{18: 1}$ & $\mathrm{C}_{18: 2}$ & $\Sigma$ Poly $\mathrm{C}_{20}$ \\
\hline 0 & 1,1 & 2,9 & 4,3 & 26,2 & 4,3 & 8,1 & 27,2 & 22,6 & 3,3 \\
\hline 7 & $<1$ & $<1$ & 2,6 & 22,6 & 5,2 & 7,3 & 30,2 & 31,4 & $<1$ \\
\hline 14 & $<1$ & $<1$ & 1,8 & 21,0 & 4,6 & 7,5 & 29,7 & 35,4 & $<1$ \\
\hline 21 & $<1$ & $<1$ & 1,9 & 19,6 & 4,5 & 5,0 & 30,2 & 38,8 & $<1$ \\
\hline 28 & $<1$ & $<1$ & 1,3 & 20,0 & 4,7 & 6,1 & $\frac{3,1}{31,1}$ & 36,7 & $<1$ \\
\hline 42 & $<-1$ & $<1$ & 1,7 & 19,9 & 4,1 & 6,2 & 30,2 & 37,9 & $<1$ \\
\hline \hline
\end{tabular}

TABLEAU 6

Evolution de la composition en acides gras des lipides corporels des rats ayant ingéré le régime C "Beurre de cacao"

(p. I00 des esters méthyliques dosés)

\begin{tabular}{c|c|c|c|c|c|c|c|c|c}
\hline$t(\mathrm{j})$ & $\mathrm{C}_{10}$ & $\mathrm{C}_{12}$ & $\mathrm{C}_{14}$ & $\mathrm{C}_{16}$ & $\mathrm{C}_{16: 1}$ & $\mathrm{C}_{18}$ & $\mathrm{C}_{18: 1}$ & $\mathrm{C}_{18: 2}$ & $\Sigma$ Poly $\mathrm{C}_{20}$ \\
\hline 0 & 1,1 & 2,9 & 4,3 & 26,2 & 4,3 & 8,1 & 27,2 & 22,6 & 3,3 \\
\hline 7 & $<1$ & $<1$ & 1,8 & 27,8 & 4,8 & 11,8 & 44,9 & 7,5 & 1,4 \\
\hline 14 & $<1$ & $<1$ & 1,9 & 27,5 & 5,4 & 12,0 & 46,3 & 5,4 & 1,5 \\
\hline 21 & $<1$ & $<1$ & 1,2 & 20,2 & 3,7 & 10,5 & 57,2 & 5,6 & 1,6 \\
\hline 28 & $<1$ & $<1$ & 1,3 & 23,5 & 4,2 & 10,9 & 53,7 & 5,1 & 1,3 \\
\hline 42 & $<1$ & $<1$ & 1,4 & 22,9 & 4,0 & 11,6 & 52,9 & 5,3 & 2,5 \\
\hline \hline
\end{tabular}

Ainsi, entre le $7^{\mathrm{e}}$ et le $42^{\mathrm{e}}$ jour, dans le lot " $\mathrm{C}$ " la concentration en acide oléique $\left(C_{18: 1}\right)$ augmente de 8 p. Ioo alors que celle en acide palmitique $\left(C_{16: 0}\right)$ baisse de 5.p roo. Dans le lot " $T$ " au cours de la même période, la concentration en acide lino- 
léique $\left(\mathrm{C}_{18: 8}\right)$ augmente de 6 p. Ioo tandis que celle en acide palmitique baisse de 3 p. Ioo.

Dans nos conditions expérimentales et après $I_{5}$ jours de mise au régime dans le cas d'ingestion d'huile de tournesol ou $2 \mathbf{I}$ jours en cas d'ingestion de beurre de cacao, la composition en acides gras du tissu adipeux peut être considérée comme étant stabilisée.

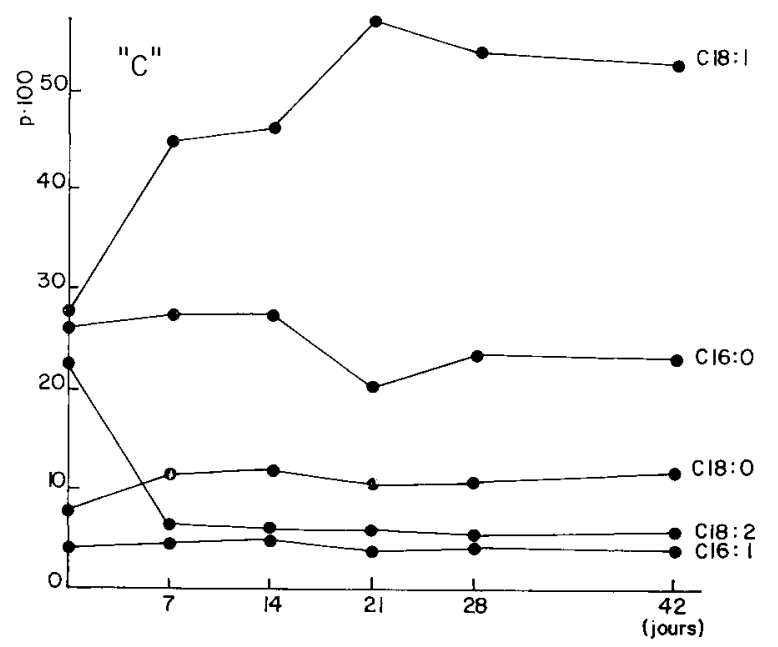

Graph. I. - Evolution de la composition en acides gras des réserves corporelles en cas d'ingestion de beurve de cacao et en fonction du temps

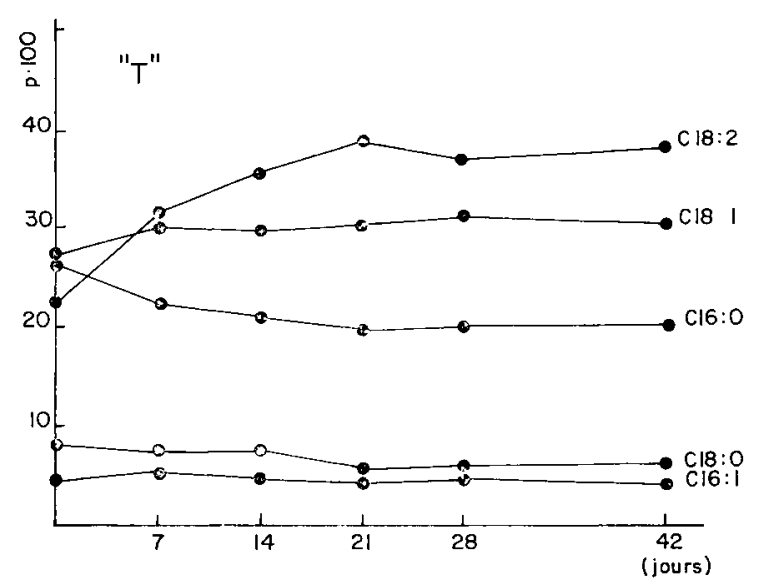

GrapH. 2. - Evolution de la composition en acides gras des réserves corporelles en cas d'ingestion d'huile de tournesol et en fonction du temps

\section{2. - Influence du type de lipide absorbé}

sur la composition finale des réserves adipeuses en acides gras

L'absorption régulière pendant 42 jours d'un mélange d'acides gras comportant plus de $90 \mathrm{p}$. Ioo d'acides gras longs et insaturés (T) donne lieu à la formation d'un tissu adipeux contenant $75 \mathrm{p}$. Ioo d'acides gras longs et insaturés $\left(\geqslant \mathrm{C}_{18: 1}\right)$. Pendant 
la même période, l'absorption régulière d'une quantité identique d'un mélange d'acides gras comportant cette fois 60 p. Ioo d'acides gras longs et saturés (C) donne lieu à la formation d'un tissu adipeux contenant 67 p. Ioo d'acides gras longs et insaturés. Sur le tableau 7 , on peut remarquer que dans le lot " $C$ ", pour une absorption d'acides gras longs et saturés pourtant 5 fois plus importante que dans le lot $T$, on obtient au niveau du tissu adipeux la mise en réserve d'une quantité de ces acides gras seulement $\mathrm{I}, 3$ fois plus importante que dans le lot « $T$ ». En cas de fort apport d'acides gras saturés et longs $\left(\mathrm{C}_{10: 0}\right.$ et $\left.\mathrm{C}_{18: 0}\right)$ ou en cas de fort apport d'acides gras longs mono- et polyinsaturés, et dans nos conditions expérimentales, on ne modifie donc que très peu le rapport acides gras saturés/acides gras insaturés dans la carcasse. En revanche, on modifie sérieusement le rapport acide oléique déposé/ $\Sigma$ des acides gras insaturés déposés (99,0 p. roo dans le lot « $C$ ") contre 43,o p. Ioo dans le lot ( $T »)$.

TABLEAU 7

Quantités globales d'acides gras saturés et insaturés absorbés et déposés (bilan réalisé sur 28 jours d'expérimentation)

\begin{tabular}{|c|c|c|c|c|}
\hline Régimes & Acides gras & $\begin{array}{c}\text { Saturés } \\
\left(\mathrm{C}_{16: 0}+\mathrm{C}_{18: 0}\right)\end{array}$ & $\begin{array}{c}\text { Insaturés } \\
\left(\mathrm{C}_{16: 1}, \mathrm{C}_{18: 1}, \mathrm{C}_{18: 2}\right)\end{array}$ & $\frac{\sum \text { saturés }}{\sum \text { insaturés }}$ \\
\hline \multirow{2}{*}{$\mathrm{C}$} & Absorbés & 15,64 & 10,77 & 1,45 \\
\hline & Déposés & 3,67 & 7,33 & 0,50 \\
\hline \multirow{2}{*}{$\mathrm{T}$} & Absorbés & 3,61 & 24,15 & 0,15 \\
\hline & Déposés & 2,73 & 8,42 & 0,32 \\
\hline
\end{tabular}

L'ingestion d'une forte quantité d'acide palmitique $\left(\mathrm{C}_{\mathbf{1 6}: 0}\right)$ et d'acide stéarique $\left(\mathrm{C}_{18: 0}\right)$ ne peut donc pas être considérée comme étant un facteur favorisant, chez le jeune Rat, la mise en place d'un tissu adipeux riche en acides gras saturés.

\section{3. - Variations de la composition en acides gras des lipides totaux hépatiques}

L'évolution des poids et des teneurs en lipides totaux des foies en fonction du temps est identique dans les deux lots. De o à 42 jours, le poids de l'organe passe de $2,4 \mathrm{~g}$ à $5,6 \mathrm{~g}$ tandis que la teneur en lipides (par rapport au poids frais) passe de 4,2 à environ 5 p. Ioo (tabl. 8).

Les tableaux 9 et ro rapportent l'évolution de la composition en acides gras des lipides totaux hépatiques. Sept jours après la mise aux régimes expérimentaux, les compositions se différencient nettement entre les lots " $\mathrm{C}$ " et " $\mathrm{T}$ ". Les différences se situent principalement au niveau des concentrations respectives en acides oléique $\left(\mathrm{C}_{18: 1}\right)$, linoléique $\left(\mathrm{C}_{18: 2}\right)$ et polyinsaturés en $\mathrm{C}_{20}$. Les concentrations en acide palmitique $\left(C_{16: 0}\right)$, palmitoléique $\left(C_{16: 2}\right)$ et stéarique $\left(C_{18: 0}\right)$ sont peu influencées par le type de lipide ingéré. 
TABLEAU 8

Evolution du poids du foie et de sa teneur en lipides totaux

\begin{tabular}{c|c|c|c|c|c|c}
\hline \hline Variables & Régimes & $0 \mathrm{j}$ & $7 \mathrm{j}$ & $1 / \mathrm{j}$ & $21 \mathrm{j}$ & $28 \mathrm{j}$ \\
\hline Poids du foie (g) & $\mathrm{T}$ & 2,4 & 3,4 & 4,3 & 5,2 & 5,5 \\
\hline $\mathrm{C}$ & 2,4 & 3,5 & 4,6 & 5,3 & 5,7 \\
\hline $\begin{array}{c}\text { Teneurs en lipides } \\
\text { totaux (\%) }\end{array}$ & $\mathrm{T}$ & $\mathrm{ND}$ & 4,3 & 4,3 & 4,4 & 4,8 \\
\hline $\mathrm{C}$ & $\mathrm{ND}$ & 4,2 & 4,8 & 4,6 & 5,0 \\
\hline
\end{tabular}

* ND : Non déterminée.

\section{TABLEAU 9}

Régime $C$ : Composition en acides gras des lipides totaux hépatiques (p. Ioo des esters méthyliques dosés)

\begin{tabular}{c|c|c|c|c|c|c}
\hline$t(\mathrm{j})$ & $\mathrm{C}_{16: 0}$ & $\mathrm{C}_{16: 1}$ & $\mathrm{C}_{18: 0}$ & $\mathrm{C}_{18: 1}$ & $\mathrm{C}_{18: 2}$ & $\Sigma$ poly $\mathrm{C}_{20}{ }^{*}$ \\
\hline 7 & 24,3 & 2,2 & 23,8 & 21,6 & 8,7 & 19,4 \\
\hline 14 & 20,5 & 1,6 & 24,7 & 22,0 & 7,6 & 23,6 \\
\hline 21 & 20,1 & 3,1 & 24,5 & 21,2 & 7,7 & 23,4 \\
\hline 28 & 18,5 & 2,2 & 23,7 & 23,1 & 8,0 & 24,5 \\
\hline
\end{tabular}

* Principalement $C_{20}: \mathbf{s} \omega_{6}$ et $C_{20: 4 \omega_{8}}$.

TABLEAU IO

Régime T: Composition en acides gras des lipides totaux hépatiques (p. roo des esters méthyliques dosés)

\begin{tabular}{|c|c|c|c|c|c|c|}
\hline$t(\mathrm{j})$ & $C_{16: 0}$ & $C_{16: 1}$ & $C_{18: 0}$ & $\mathrm{C}_{18: 1}$ & $C_{18: 2}$ & $\Sigma$ poly $\mathrm{C}_{20}{ }^{*}$ \\
\hline 7 & 20,2 & 1,2 & 21,0 & 10,0 & 17,3 & 30,3 \\
\hline 14 & 21,9 & 1,1 & 22,7 & 9,1 & 18,9 & 31,5 \\
\hline 21 & 20,1 & 1,1 & 23,4 & 10,8 & 18,9 & 25,7 \\
\hline 28 & 20,4 & 1,1 & 22,1 & 10,2 & 19,4 & 26,8 \\
\hline
\end{tabular}

* Principalement $\mathrm{C}_{20: 3 \omega_{6}}$ et $\mathrm{C}_{20}: \omega_{6}$. 
Au fur et à mesure que se déroule l'expérience, on n'observe plus que de très légers changements dans les concentrations respectives en acides gras par rapport à ce qui est observé après 7 jours de soumission aux différents régimes.

Les teneurs en acide oléique $\left(\mathrm{C}_{18: 1}\right)$ et en acide linoléique $\left(\mathrm{C}_{18: 2}\right)$ s'inversent en passant des animaux du lot " $T$ " à ceux du lot « $C$ ». Les teneurs en acides gras en $\mathrm{C}_{20}$ polyinsaturés sont nettement plus élevées en début d'expérience dans les foies des jeunes animaux recevant le régime ' $\mathrm{T}$. Par la suite, le phénomène s'estompe. La concentration totale en acides gras longs et saturés peut être considérée comme restant identique dans les 2 types de tissu hépatique.

\section{4. - Observations morphologiques}

Sur les coupes semi-fines de tissu adipeux mésentérique des rats, sacrifiés au moment de la mise en expérience, l'aspect topographique de la répartition des différents éléments de ce tisstu a été observé en microscopie photonique. L'importance quantitative des cellules avec inclusions lipidiques et des cellules sans lipides a été appréciée en vue de leur comparaison avec les tissus correspondants des rats soumis aux régimes " $\mathrm{C}$ " et " $\mathrm{T}$ ).

Sur les coupes fines des mêmes tissus observées en microscopie électronique (fig. I), on note que les cellules adipeuses, dont les lipides ont déjà fusionné en une seule masse, c'est-à-dire les cellules que nous appellerons les adipocytes, sont relativement nombreuses mais que leur répartition est très variable suivant le niveau et surtout en fonction de l'emplacement des vaisseaux sanguins dont certains sont en formation. D'une façon générale, on peut dire que les cellules les plus jeunes sont toujours au voisinage de la paroi vasculaire. Les préadipocytes avec un nombre variable d'inclusions lipidiques et les préadipocytes sans lipides sont, à cet âge, les éléments prédominants.

Sur les coupes semi-fines des tissus adipeux mésentériques des rats des lots " $\mathrm{C}$ " et " $T$ ", sacrifiés après 7 jours de régime (fig. 2 et 3 ), l'aspect structural observé en microscopie photonique est identique pour les tissus des rats des deux régimes et ne varie pas sensiblement comparé à celui des tissus prélevés sur les rats au début de l'expérience (fig. I).

L'observation des coupes effectuées en microscopie électronique ne permet pas de mettre en évidence la moindre différence structurale ou ultrastructurale entre les tissus adipeux mésentériques prélevés sur les rats des lots " $\mathrm{C}$ » et " $\mathrm{T}$ " (fig. 2 et 3 ). I'image de ces coupes est assez semblable à ce qui a été observé au début de l'expérience, néanmoins il apparaît que le nombre des adipocytes par rapport aux préadipocytes avec et sans inclusions lipidiques est inférieur après 7 jours d'expérience à ce qu'il était au début de celle-ci. Deux hypothèses sont possibles : les adipocytes ont perdu une partie de leurs lipides et, comme dans le cas d'un amaigrissement, sont revenus à un stade antérieur de leur évolution qui est celui de préadipocyte avec un nombre variable d'inclusions lipidiques ou bien, et cela nous semble concorder avec les analyses biochimiques, le nombre des préadipocytes, dont la prolifération est importante à cet âge, augmente sensiblement, ce qui ralentit le passage du stade préadipocyte au stade adipocyte aussi bien pour le régime " $\mathrm{C}$ » que pour le régime " $T$ ).

Les adipocytes des deux régimes sont à des stades variables de maturation, 


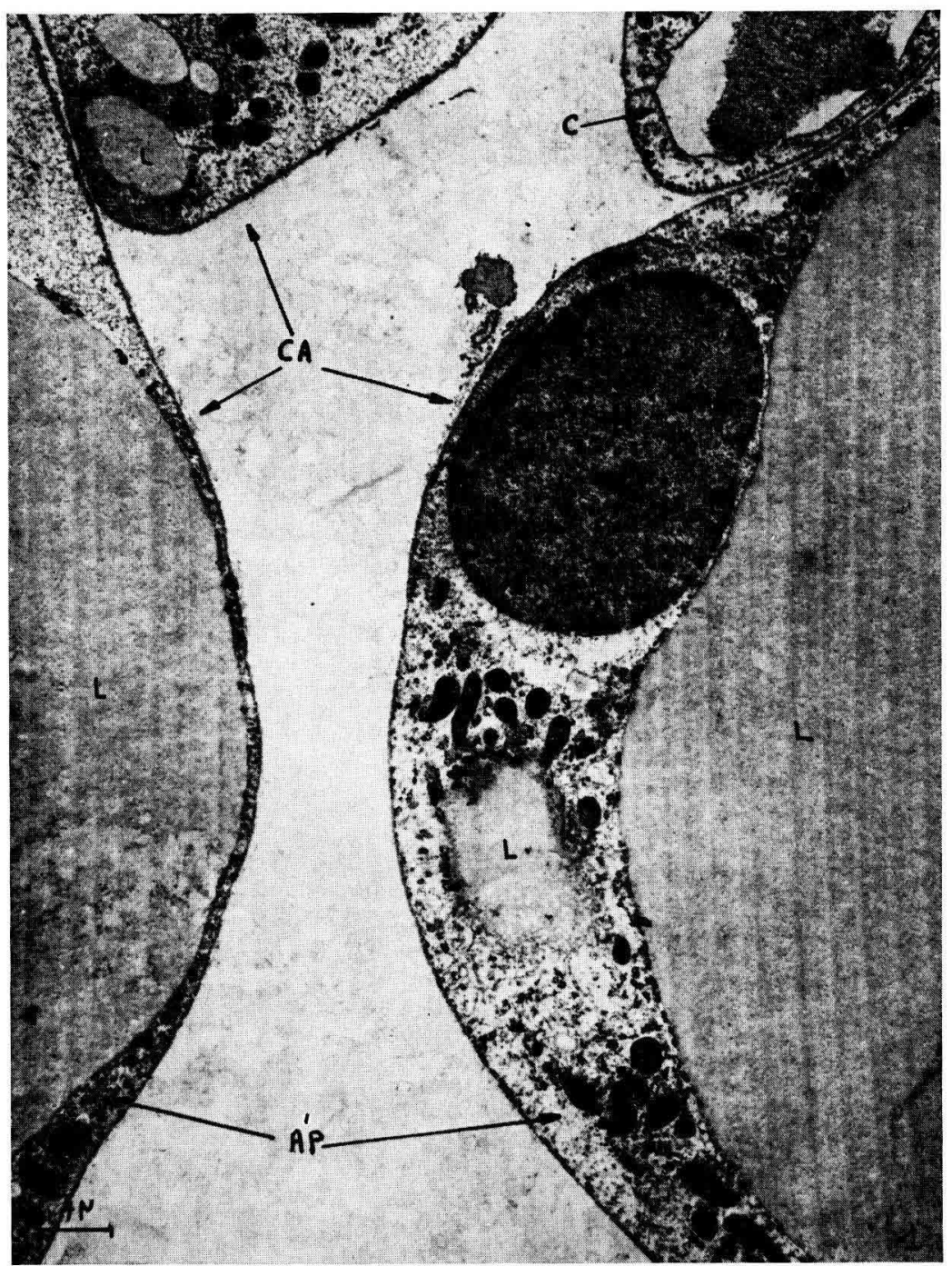

FIG. I. - Vue partielle d'une coupe de 3 cellules de tissu adipeux mésentérique prélevé sur un des rats sacrifiés au moment de la mise aux régimes " $C$ " et " $T$ " des autres animaux appartenant au même lot.

Anneau périphérique : AP ; capillaire sanguin : $\mathrm{C}$; cellule adipeuse : $\mathrm{CA}$; lipides : $\mathrm{L}$; noyau : $\mathrm{N}$. 


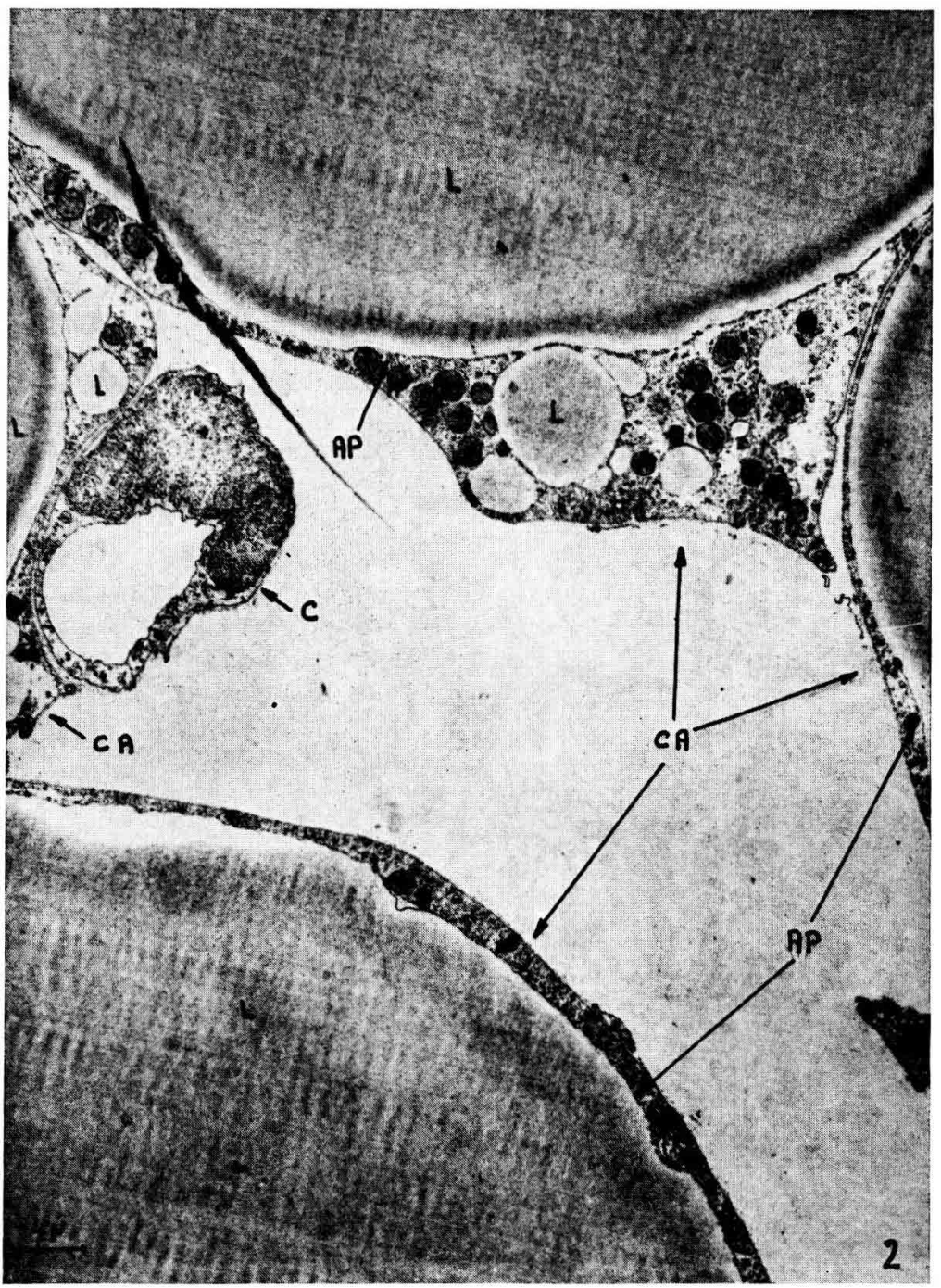

FIG. 2. - Vue partielle d'une coupe de 4 cellules de tissu adipeux mésentérique prélevé sur un des rats ayant ingéré pendant 7 jours de l'huile de tournesol. Dans ce cas, la masse lipidique est toujours caractérisée par une bordure très claire en contact avec le cytoplasme et par une zone très contrastée à la périphérie de la masse lipidique.

Anneau périphérique : AP ; capillaire sanguin: $\mathrm{C}$; cellule adipeuse : $\mathrm{CA}$; lipides : $\mathrm{L}$; mitochondries : $M$. 


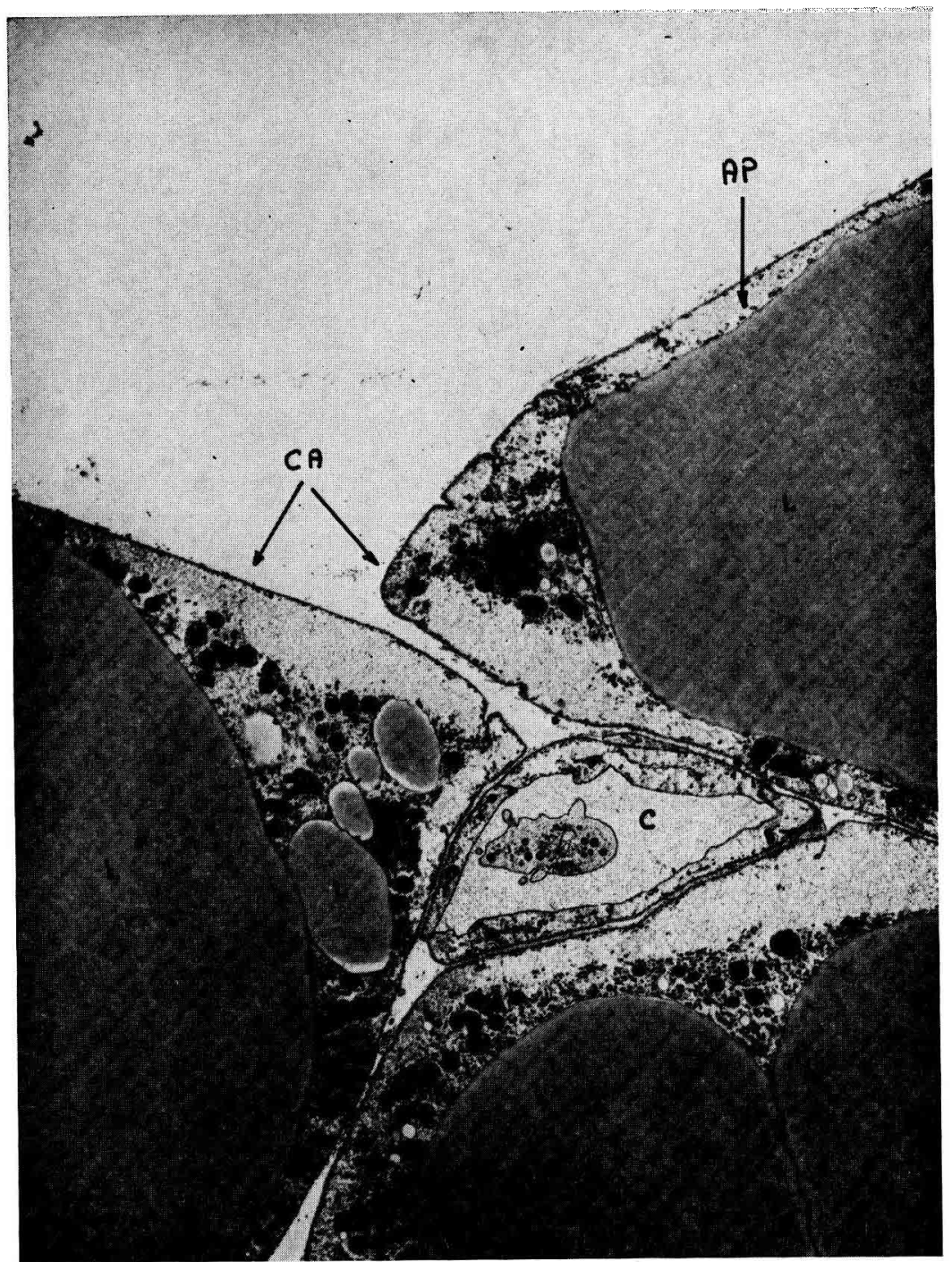

FIG. 3. - - Vue partielle d'une coupe de 3 cellules de tissu adipeux mésentérique prélevé sur un des rats ayant ingêré du beurye de cacao pendant 7 jours. Les lipides sont homogènes, la matrice fondamentale du cytoplasme est éclaircie et les mitochondries sont très contrastées.

Anneau périphérique : AP ; capillaire sanguiı : $\mathrm{C}$; cellule adipeuse : CA ; lipides : $L$; mitochondries : $M$. 


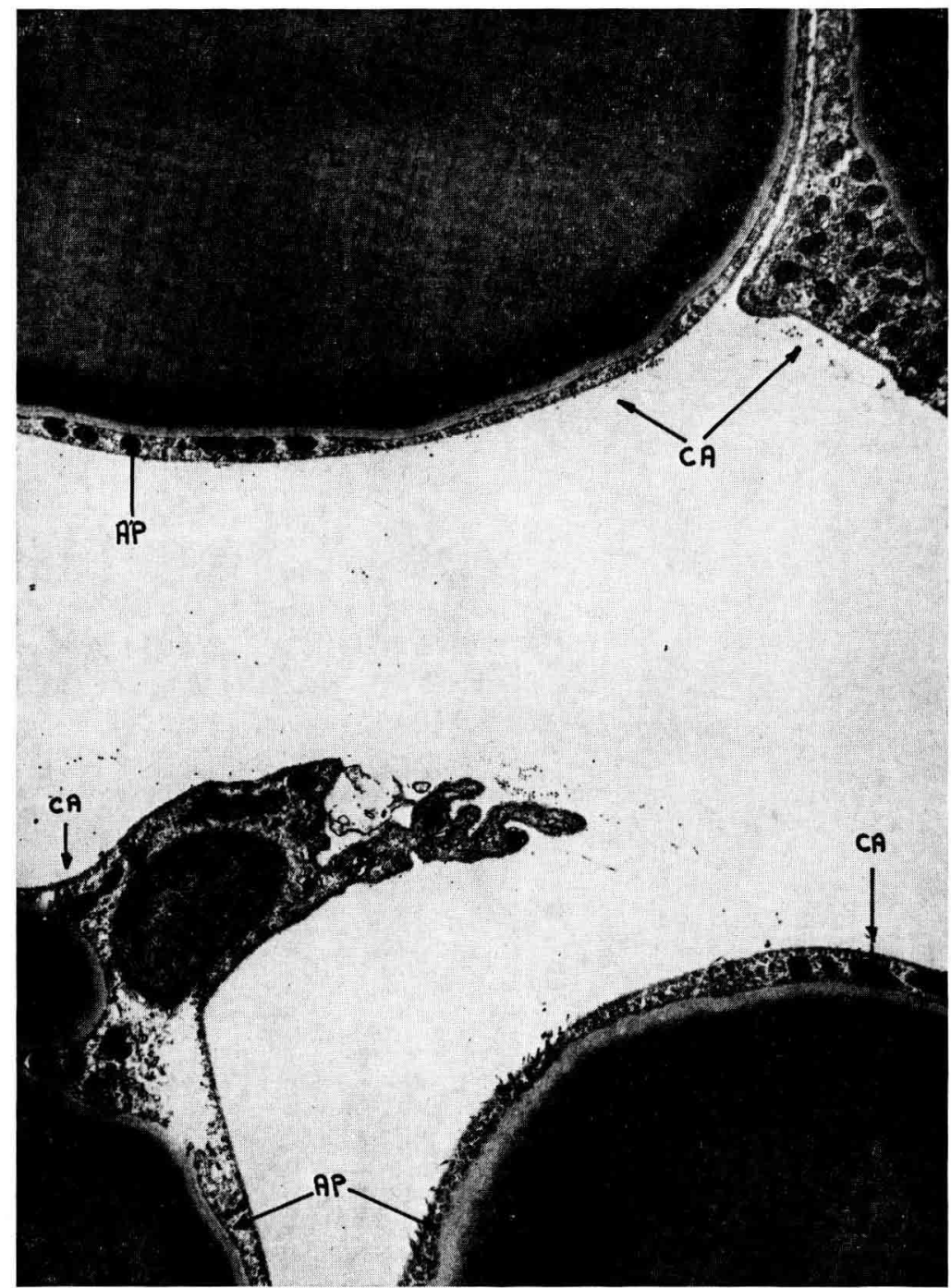

Fig. 4. - Vue partielle d'une coupe de 4 cellules de tissu adipeux mésentérique prélevé sur un des rats ayant ingêré de l'huile de tournesol pendant 35 jours. L'aspect des lipides est semblable, avec plus de contraste, à ce qui a été observé sur la figure 2 .

Anneau périphérique : AP ; cellule adipeuse : CA ; lipides : L ; mitochondries : $\mathrm{M}$; noyau : $\mathrm{N}$. 


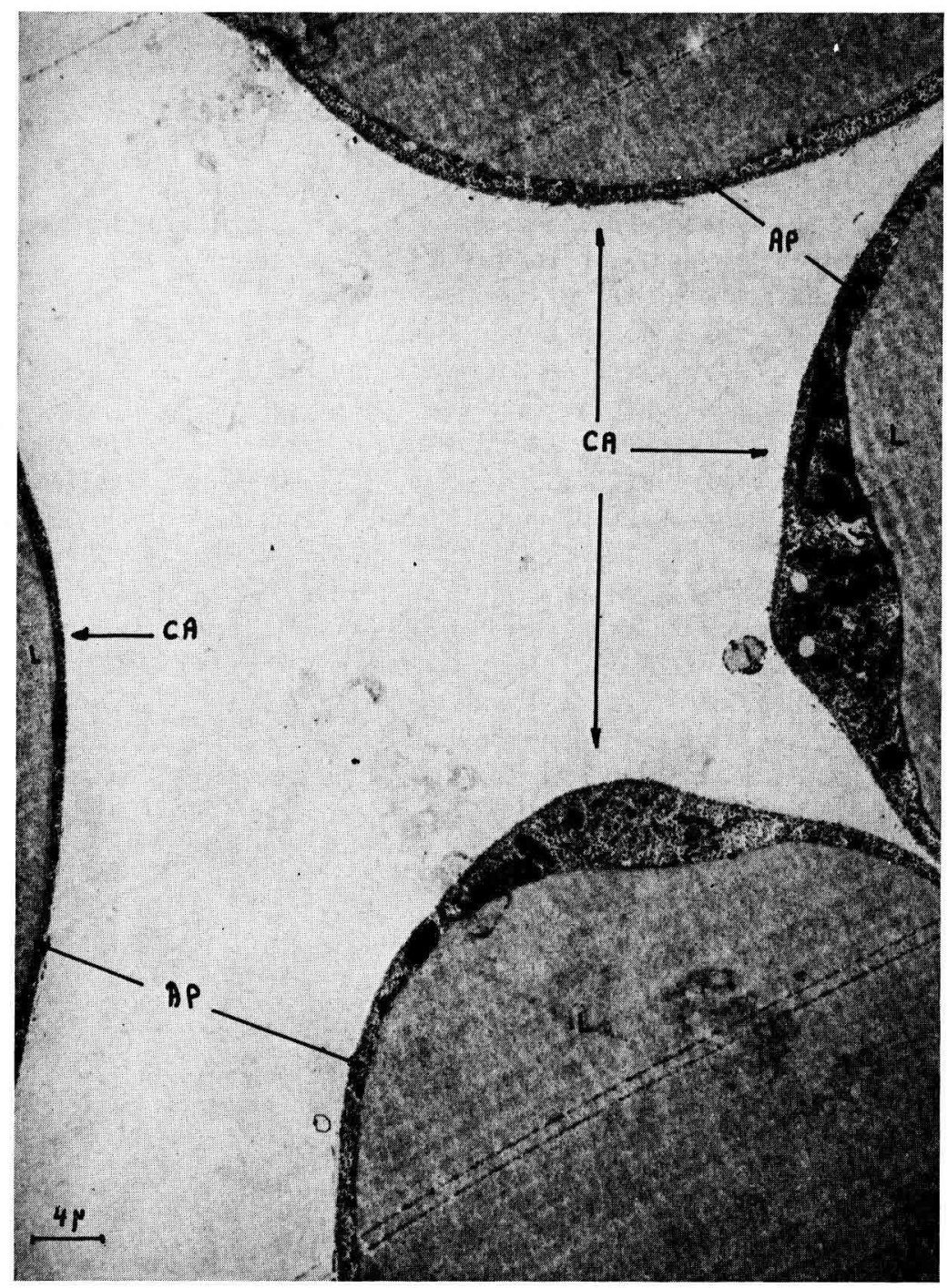

FIG. 5. - Vue partielle d'une coupe de 4 cellules de tissu adipeux mésentérique prélevé sur un des rats ayant ingéré du beurre de cacao pendant 35 jours.

Anneau périphérique : AP ; cellule adipeuse : CA ; lipides : L ; mitochondries : M. 
cependant l'anneau périphérique de la majorité de ces cellules est important et bien conservé. La lame basale et la membrane plasmique sont en bon état, ce qui indique une activité normale de ces cellules. La matrice fondamentale du cytoplasme, bien qu'elle soit comprimée par les inclusions lipidiques, est généralement d'aspect normal et de contraste moyen. Les mitochondries sont d'aspect normal, leur nombre est dans la plupart des adipocytes encore important et leur répartition dans l'anneau périphérique est généralement régulière. Les adipocytes parvenus au stade terminal de leur évolution sont rarement observés. La taille de ces cellules est très variable, leur diamètre pouvant être le plus souvent estimé entre 50 et $90 \mu$.

Les préadipocytes avec des inclusions lipidiques sont aux différents stades de leur évolution et le nombre des préadipocytes sans lipides et des capillaires sanguins en formation est relativement important. On peut donc dire que ces tissus correspondent bien par leur aspect à l'âge de croissance des rats mais du fait que l'on observe des cellules à tous les degrés d'évolution, que les cellules sont bien individualisées et qu'elles sont entourées par une lame basale très apparente, on peut penser que le développement de ces tissus est un peu ralenti.

I, 'aspect structural et ultrastructural des tissus adipeux prélevés après $\mathrm{I}_{5}, 2 \mathrm{I}$ et 28 jours de régime sur les rats des lots " $C$ " et " $T$ ” est toujours s smblable pour les deux lots, de même l'évolution et le rapport des différents éléments de ces tissus ne change que peu.

Pour les rats sacrifiés à 35 jours (fig. 4 et 5 ) et surtout à 42 jours de régime, l'aspect des tissus est le même dans les deux lots mais le rapport entre adipocytes et préadipocytes semble évoluer à l'avantage des premiers ; cela peut être interprété par un ralentissement dans l'apparition de nouvelles cellules. On observe d'autre part un nombre non négligeable d'adipocytes pour lesquels, si on juge leur aspect structural et ultrastructural, la mobilisation des lipides est accélérée. Cela est sans doute la conséquence du mode d'alimentation, lequel ne permet pas aux animaux de stocker beaucoup de lipides.

\section{DISCUSSION ET CONCLUSIONS}

La mise en réserve des acides gras chez le jeune Rat est très fortement influencée par la qualité des lipides ingérés. Après le sevrage, même en se plaçant à des vitesses de croissance moyennes $(4 \mathrm{~g} / \mathrm{j})$ et dans des conditions d'adipogenèse modérée, l'impact des matières grasses alimentaires sur la composition en acides gras des lipides de réserve de l'animal est considérable. Cependant, ce qui semble le plus remarquable reste les modifications profondes que l'on observe à ce niveau au cours de la première semaine de mise au régime expérimental. Par la suite et très rapidement, le tissu adipeux du jeune rat présente une composition en acides gras d'une remarquable stabilité.

Les animaux utilisés au cours de cette expérimentation n'ont pas été soumis à plus de 6 semaines d'application des 2 régimes testés. Nos données complètent celles précédemment rapportées par CLÉMENT et al. (I963) qui étudiaient les modifications de la structure des triglycérides de réserve et de leur composition en acides gras en fonction de la nature des lipides ingérés et montraient une évolution de la composition 
en acides gras en fonction du temps. Leurs analyses ne commençaient cependant qu'après 7 semaines d'application de régimes contenant soit du saindoux, soit de l'huile de tournesol. Notre travail permet d'observer ce qui se passe durant la période antérieure.

Si l'on associe les résultats de ces 2 travaux différents, on peut dire qu'après le sevrage, la composition en acides gras des réserves adipeuses se modifie profondément selon le type de lipide ingéré et qu'un équilibre est rapidement atteint et se maintient sans doute jusqu'à ce que l'augmentation de poids ne se fasse plus principalement que par une augmentation de la masse adipeuse. A partir de ce moment, on peut penser que l'impact des lipides exogènes se fait plus fortement sentir et que la composition en acides gras tend à évoluer de plus en plus vers celle qui est caractéristique des lipides ingérés.

Chez le jeune Rat, l'absorption d'une forte quantité d'acides gras longs et saturés $\left(\mathrm{C}_{16: 0}\right.$ et $\left.\mathrm{C}_{18: 0}\right)$ ne donne pas lieu à l'édification concomitante d'un tissu adipeux riche en acides saturés. On assiste plutôt à la mise en réserve d'une quantité très importante d'acides gras longs mono-insaturés $\left(\mathrm{C}_{18: 1}\right.$ principalement). L,es teneurs en acides gras longs saturés ne sont que très faiblement supérieures à celles qui sont enregistrées en cas d'ingestion de quantités identiques de lipides très insaturés (Huile de tournesol) et ne sont pas en rapport avec les très fortes quantités absorbées. Les concentrations en acide oléique ( 45 à $50 \mathrm{p}$. IOO) sont de plus peu différentes de celles enregistrées par Bolirnger (I963) ou CLÉMENT et al. (Ig63) en cas d'ingestion d'un régime lipidoprive ou en cas d'ingestion de tricapryline.

D'après les résultats obtenus ici, il semblerait bien qu'il existe un mécanisme régulateur dont le but serait d'équilibrer les synthèses et les transformations de façon à conserver le rapport $\Sigma$ acides gras saturés $/ \Sigma$ acides gras insaturés dans la carcasse le plus proche possible de celui qui est caractéristique de l'espèce animale étudiée.

Le rôle " tampon " semble être tenu par l'acide oléique $\left(C_{18: 1}\right)$ dont la biosynthèse ou/et la mise en réserve est favorisée en cas d'un fort apport exogène d'acides gras longs saturés, et se trouve être fortement réprimée en cas d'un fort apport d'acide linoléique $\left(\mathrm{C}_{18: 2}\right)$.

D'après les données de BOLLINGER (I963) pour un apport calorique identique sous forme de différents triglycérides homogènes, on trouve dans les réserves adipeuses :

- 68 p. Ioo d'acides gras insaturés en cas d'ingestion de Trilinoléine;

- 77 p. Ioo d'acides gras insaturés en cas d'ingestion de Trioléine;

- 55 p. Ioo d'acides gras insaturés en cas d'ingestion de Tripalmitine;

- 48 p. Ioo d'acides gras insaturés en cas d'ingestion de Trimyristine;

- 63 p. Ioo d'acides gras insaturés en cas d'ingestion de Tricapryline;

- 66 p. roo d'acides gras insaturés en cas d'ingestion d'un régime lipidoprive.

Nos données jointes à celles de BOLLINGER démontrent donc que pour obtenir les dépôts adipeux les plus saturés chez le Rat, il faut éliminer les chaînes longues insaturées et saturées (surtout le $\mathrm{C}_{18}: 0$ ) ainsi que les chaînes courtes $\left(\leqslant \mathrm{C}_{10}\right)$. Les meilleurs résultats semblent donc être ceux obtenus par cet auteur avec des chaînes saturées comme l'acide myristique $\left(\mathrm{C}_{14: 0}\right)$ et ceci confirme également les résultats obtenus dans d'autres espèces et plus particulièrement chez le Porc par FI,ANZy et al. (I970) et Peraza (I972) quand on emploie l'acide laurique ( $\left.\mathrm{C}_{12: 0}\right)$. 
Au niveau de la composition en acides gras des lipides hépatiques, on observe également une influence très nette de la nature des lipides ingérés. Comme pour ce qui est observé pour le cas des lipides de la carcasse, le bouleversement de la composition en acides gras après la soumission aux 2 régimes expérimentaux est immédiat. On note également que les teneurs en acides gras polyinsaturés en $C_{20} \quad\left(C_{20}::_{3} \omega_{6}\right.$ et $\mathrm{C}_{20: 4 \omega_{6}}$ ) provenant de l'élongation et de la désaturation de l'acide linoléique $\left(\mathrm{C}_{18: 2 \omega_{6}}\right)$ sont très voisines dans les 2 lots au bout de 4 semaines. Compte tenu du fait que cet acide est nécessairement d'origine exogène et que le régime " $C$ " en apporte I5 fois moins que le régime " $T$ ", on conçoit aisément que dans le lot " $C$ " presque tout l'apport alimentaire ait été " bloqué " au niveau du foie afin d'assurer la synthèse des homologues supérieurs et que la mise en réserve de cet acide dans la carcasse ait été très basse.

Les données microscopiques confirment le fait qu'au cours de cette expérimention l'adipogenèse a été lente et que la structure histologique du tissu adipeux a évolué lentement. Ceci est en relation avec le mode d'alimentation particulier qui a du être adopté et qui n'a permis qu'une croissance quotidienne de 4 g/jour alors qu'en alimentation ad libitum, avec des régimes similaires, des vitesses de croissance de $6 \mathrm{~g} /$ jour sont couramment enregistrées au laboratoire.

Dans ces conditions nutritionnelles bien particulières, qui permettent d'observer une évolution lente des adipocytes, on ne note pas de différences histologiques fondamentales entre les tissus adipeux mésentériques provenant d'animaux ayant reçu dans leur ration soit un fort pourcentage d'acides gras longs et insaturés $\left(\mathrm{C}_{18: 1}\right.$ et $\left.\mathrm{C}_{18: 2}\right)$, soit un fort pourcentage d'acides gras longs et saturés $\left(\mathrm{C}_{16: 0}\right.$ et $\left.\mathrm{C}_{18: 0}\right)$.

Rę̧u pour publication en avril 1974 .

\section{REMERCIEMEN'TS}

Ce travail a bénéficié d'une aide financière dans le cadre de l'action thématique programmée I. N. S. E. R. M. no I9 "Lipogenèse chez les mammifères".

\section{SUMMARY}

\section{EVOLUTION OF LIPID STORES IN THE YOUNG GROWING RAT IN RELATION TO TIME AND QUALITY OF LIPIDS INGESTED}

Total lipid content, and fatty acid composition of lipids, carcasses and livers, as well as the histological structure of mesenteric adipose tissue, are followed for 6 weeks in 2 lots of recently weaned young rats. The animals are fed the same basic diet and absorb isoenergetic quantities of lipids containing either 70 p. I oo saturated long fatty acids (as much $\mathrm{C}_{16: 0}$ as $\mathrm{C}_{18: 0}$ ) or 85 p. Ioo unsaturated and long fatty acids $\left(\mathrm{C}_{18: 1}\right.$ and $\left.\mathrm{C}_{18: 2}\right)$.

In the experimental conditions explained in this text, growth, adipogenesis and proteinogenesis are identical in the 2 lots.

Fatty acid composition of body reserves, as well as of hepatic lipids, is totally modified after 7 days on the two experimental diets, and the fatty acid compositions are considered as completely stabilized after $2 \mathrm{I}$ days. Absorption of saturated and long fatty acids $\left(\mathrm{C}_{16: 0}\right.$ and $\left.\mathrm{C}_{1 \mathrm{~B}: 0}\right)$ in 
body lipids favorizes the storage of a large quantity of mono-saturated fatty acids, and mainly oleic acid $\left(\mathrm{C}_{18: 1}\right)$.

When there is a large contribution of exogenous saturated and long fatty acids (as much $\mathrm{C}_{18: 0}$ as $\left.\mathrm{C}_{18: 0}\right)$ or of long mono and poly-unsaturated fatty acids $\left(\mathrm{C}_{18: 1}\right.$ and $\left.\mathrm{C}_{18: 2}\right)$, the ratio $\Sigma$ saturated fatty acids $/ \Sigma$ unsaturated fatty acids is little changed. However, the ratio oleic acid deposited $/ \Sigma$ of unsaturated fatty acids deposited is seriously modified. These results emphasize the role of oleic acid in regulating the ratio $\Sigma$ saturated fatty acids $/ \Sigma$ unsaturated fatty acids in relation to the type of lipid ingested.

Histological studies show that in our feeding and growth conditions up to about the age of 70 days, there is no morphological difference observed between the two types of mesenteric adipose tissue.

\section{RÉFÉRENCES BIBLIOGRAPHIQUES}

Bollinger J. N., I963. The metabolism of fatty acids derived from dietary triglycerides. Thesis, Texas A. and M. University, $76 \mathrm{p}$.

Carroll K. K., 1965. Dietary fat and the fatty acid composition of tissue lipids. J. Am. Oil. Chem. Soc., 42,5 I $6-528$.

Clément J., Boucrot P., Loriette C., Raulin J., I963. Influence de la nature des lipides alimentaires sur la composition et la structure des triglycérides de réserve du Rat blanc. Bull. Soc. Chim. Biol., 45, IO3I-I042.

Flanzy J, François A. C., Rérat A., 1970. Utilisation métabolique des acides gras chez le Porc. Ann. Biol. anim. Bioch. Biophys., 10, 603-620.

Folch J., Lees M., Slone-Stanley G. H., 1957. A simple method for the isolation and purification of total lipids from animal tissues. J. Biol. Chem., 226, 497-509.

Kirschner S. L., Harris R. S., I96r. The effect of chain length on the metabolism of saturated fatty acids by the Rat. J. Nutr., 73, 397-402.

LeEson C. R., LeEson T. S., I970. Staining methods for sections of epon-embedded tissues for light microscopy. Can. J. Zool., 48, 189-19o.

Millonig G., I96r. The advantages of phosphate buffer for $\mathrm{O}_{3} \mathrm{O}_{4}$ solutions in fixation. J.appl. Phys., 32, r637.

PERAZA C. F., I972. Utilisation énergétique comparée de la trilaurine et d'huiles végétales insaturées par le Porc. Thèse Doctorat Université, Paris VI, 59 p.

Sмith S., Watts R., Dils R., I968. Quantitative gas-liquid chromatographic analysis of rodent milk triglycerides. J. Lipid Res., 9, 52-57.

Tove S. B., Smith F. H., I960. Changes in the fatty acid composition of the depot fat of mice induced by feeding oleate and linoleate. J. Nutr., $\mathbf{y} 1,264-272$. 